Fabrication of quantum point contacts by engraving GaAs/AlGaAs heterostructures with a diamond tip

J. Regul, U. F. Keyser, M. Paesler, F. Hohls, U. Zeitler, R. J. Haug, A. Malavé, E. Oesterschulze, D. Reuter, and A. D. Wieck

Citation: Appl. Phys. Lett. 81, 2023 (2002); doi: 10.1063/1.1506417

View online: https://doi.org/10.1063/1.1506417

View Table of Contents: http://aip.scitation.org/toc/apl/81/11

Published by the American Institute of Physics

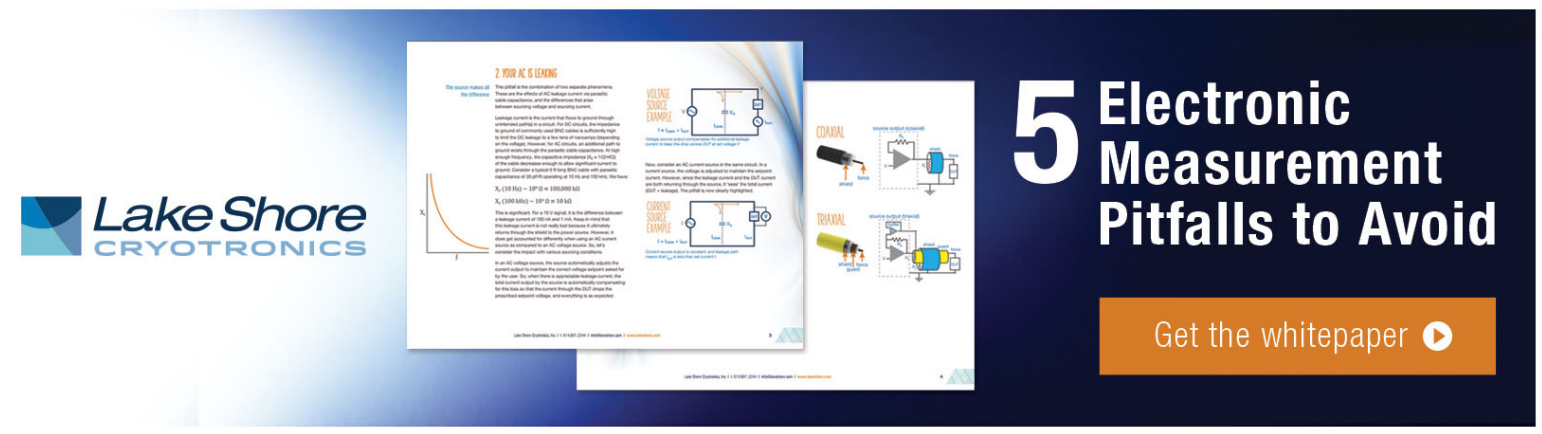




\title{
Fabrication of quantum point contacts by engraving GaAs/AIGaAs heterostructures with a diamond tip
}

\author{
J. Regul, U. F. Keyser, ${ }^{\text {a) }}$ M. Paesler, F. Hohls, U. Zeitler, and R. J. Haug \\ Institut für Festkörperphysik, Universität Hannover, 30167 Hannover, Germany \\ A. Malavé and E. Oesterschulze \\ Institut für Technische Physik, Universität Kassel, 34132 Kassel, Germany \\ D. Reuter and A. D. Wieck \\ Lehrstuhl für Angewandte Physik, Ruhr-Universität Bochum, 44780 Bochum, Germany
}

(Received 25 February 2002; accepted for publication 17 July 2002)

\begin{abstract}
We use the all-diamond tip of an atomic force microscope for the direct engraving of high-quality quantum point contacts in GaAs/AlGaAs heterostructures. The processing time is shortened by two orders of magnitude compared to standard silicon tips. Together with a reduction of the line width to below $90 \mathrm{~nm}$, the depletion length of insulating lines is reduced by a factor of two with the diamond probes. The such fabricated defect-free ballistic constrictions show well-resolved conductance plateaus and the 0.7 anomaly in electronic transport measurements. (c) 2002 American Institute of Physics. [DOI: 10.1063/1.1506417]
\end{abstract}

Over the last few years the atomic force microscope (AFM) has been used as a flexible nanolithographic tool for the direct patterning of surfaces. ${ }^{1}$ It offers not only a convenient and simple way to fabricate submicron devices but also permits in situ control of relevant sample parameters during the lithography process. ${ }^{2}$ A successful and straightforward method is the mechanical manipulation of semiconductors surfaces by means of an AFM tip. The feasibility of this technique has been demonstrated for various materials like $\mathrm{GaSb},{ }^{3} \mathrm{InAs},{ }^{4}$ and GaAs. ${ }^{2,5}$

Here we present the application of the engraving technique to fabricate quantum point contact devices in GaAs/ AlGaAs heterostructures. We show that new all-diamond tips are ideally suitable for the manufacturing of defect-free ballistic channels in two-dimensional electron gases. We consider the importance of the AFM-tip material by comparing the device properties of samples patterned by a silicon tip and by a diamond tip. Because of its highest possible Mohs hardness of 10 diamond represents the ideal tip material for the engraving. For the patterning we use standard silicon tips $^{6}$ and all-diamond AFM tips with force constants of more than $40 \mathrm{~N} / \mathrm{m}$. The latter were grown by hot-filament chemical vapor deposition of polycrystalline diamond onto a prepatterned silicon substrate. Details on the fabrication technique are given in Ref. 7.

The samples presented in this experiment are based on a modulation doped GaAs/AlGaAs heterostructure containing a two-dimensional electron gas (2DEG) $57 \mathrm{~nm}$ below the sample surface with a sheet density of $4.07 \times 10^{15} \mathrm{~m}^{-2}$ and a mobility of $107 \mathrm{~m}^{2} / \mathrm{V} \mathrm{s}$, the layer sequence is shown in Fig. 1(a). We fabricated Hall bar geometries with standard photolithography, wet-chemical etching and alloyed $\mathrm{Au} / \mathrm{Ge}$ contacts. Afterwards the samples were bonded and mounted into the AFM for the controlled engraving process. For the scribing the AFM tip is repeatedly scanned over the Hall bar with

${ }^{a)}$ Electronic mail: keyser@nano.uni-hannover.de a scanning speed of $0.1 \mathrm{~mm} / \mathrm{s}$ and a contact force of several tens $\mu \mathrm{N}$. Due to this high loading force each scan removes some material of the cap layer which leads to a stepwise depletion of the underlying 2DEG. During the whole lithography procedure the sample resistance is monitored to control the fabrication progress. The total depopulation of the 2DEG is marked by an abrupt raise of the sample resistance to more than $3 \mathrm{M} \Omega$. For more details on our patterning procedure see Ref. 2.

Figure 1(b) shows an AFM image of an engraved line that was scribed with a Si tip by applying $50 \mu \mathrm{N}$ as loading force and scanning the tip $\sim 100$ times over the surface. The resulting line has a width of $250 \mathrm{~nm}$. The depth $z \sim 9 \mathrm{~nm}$ suffices for this heterostructure for the total depletion of the 2DEG underneath the groove.

We achieve much narrower lines of $90 \mathrm{~nm}$ width and the same depth $z \sim 9 \mathrm{~nm}$ by using an all-diamond probe as shown in Fig. 1(c). The displayed groove was manufactured by scanning the diamond tip once over the surface with a similar contact force as for $\mathrm{Si}$. Compared to the former results in Fig. 1(b) the engraving process for, e.g., a $100 \mu \mathrm{m}$ line is (a)

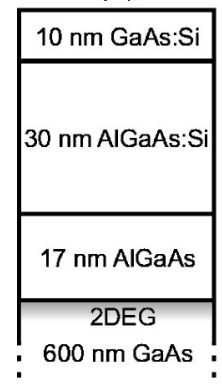

(b)

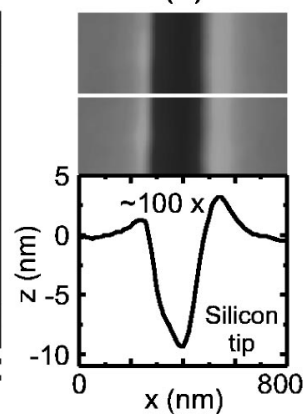

(c)

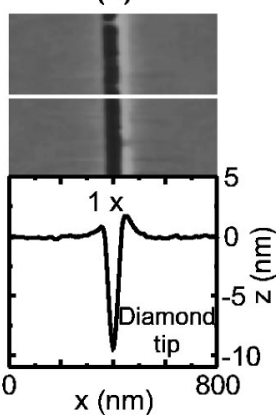

FIG. 1. (a) Layer sequence of the heterostructure. The doping concentration of the two upper layers is $\sim 0.9 \times 10^{24} \mathrm{~m}^{-3}$. (b), (c) Results of the engraving with (b) a silicon tip and (c) a diamond tip. Upper part: AFM micrograph of the grooves; lower part: depth profile along the white lines. (b) after $\sim 100$ scans with a silicon tip; (c) after one scan with a diamond tip. 

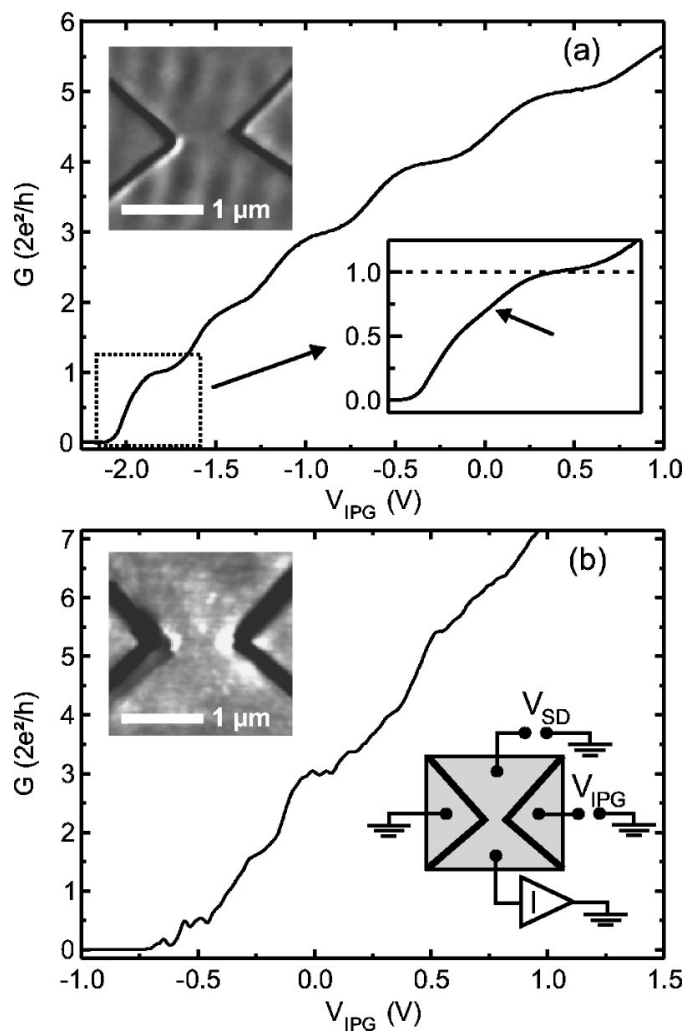

FIG. 2. Differential conductance $G\left(V_{\mathrm{IPG}}\right)=d I / d V_{\mathrm{SD}}$ in units of $2 e^{2} / h$ as a function of in-plane gate voltage. (a) Sample patterned by a diamond tip. Left inset: AFM image of a constriction formed by a diamond tip. Right inset: Magnification of the first conductance step. The arrow marks the 0.7 anomaly. (b) Silicon-patterned sample. Left inset: AFM image of a Sipatterned constriction. The schematic on the right sketches the measurement setup.

substantially reduced by nearly two orders of magnitude from minutes to a few seconds. The reduction of the line width from 250 to $90 \mathrm{~nm}$ is mainly explained by the severe tip wear of the Si tip during the writing process. After the engraving we measured the radius of the Si tip and the diamond tip by scanning electron microscopy. Whereas the Sitip radius increased by a factor of 10 to more than $100 \mathrm{~nm}$, images of the diamond tips yielded a radius of below $50 \mathrm{~nm}$ before and after the fabrication. As expected the tip wear for diamond is almost negligible. In fact, we used this diamond tip for the fabrication of more than 40 devices without any observation of tip degradation. In contrast, a silicon tip can only be utilized once in most cases.

To compare the electronic properties of the lines fabricated by the different tips we defined two 1D channels by engraving constrictions into the GaAs/AlGaAs heterostructure. The regions separated from the constriction by an insulating groove serve as in-plane gates. The upper insets of Figs. 2(a) and 2(b) show the constrictions engraved with (a) the diamond tip and (b) the Si tip. Both constrictions were electrically characterized in a pumped ${ }^{3} \mathrm{He}$-cryostat providing a base temperature of $T=350 \mathrm{mK}$.

In Fig. 2 the differential conductance $G=d I / d V_{\mathrm{SD}}$ of the diamond-patterned (a) and the silicon-patterned sample (b) is shown. For the measurement we used a standard lock-in technique at an excitation voltage of $V_{\mathrm{SD}, \text { ac }}=60 \mu \mathrm{V}$ at 13 Hz. The two conductance curves presented in Fig. 2 were recorded by varying only a single in-plane gate whereas the
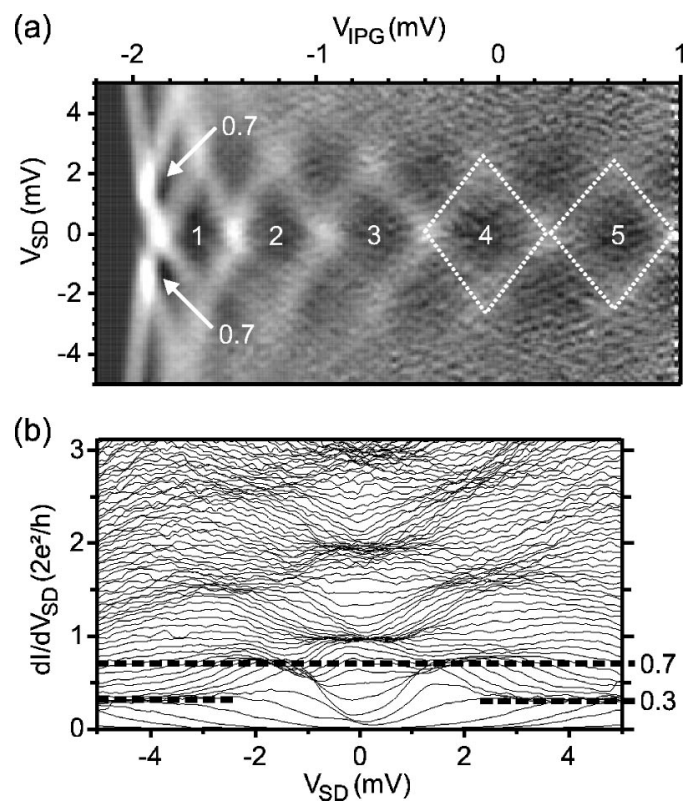

FIG. 3. (a) Gray scale plot of the transconductance $d G / d V_{\text {IPG }}$ obtained from the diamond-patterned sample at a temperature of $T=350 \mathrm{mK}$. The gate configuration is the same as in Fig. 2(b). Dark regions correspond to low transconductance (plateaus) and light regions reflect high transconductance (plateau transitions). The numbers denote the occupied subbands. The location of the 0.7 plateaus is marked by the arrows. (b) Differential conductance as a function of dc source-drain voltage taken at fixed gate voltages. The additional plateaus at 0.7 are marked by a dashed line.

second gate was kept at a fixed potential. A constant series resistance of the contacts and the 2DEG was subtracted. A schematic image of the measurement setup is shown in the lower inset of Fig. 2(b).

The curve in Fig. 2(a) corresponding to the diamondpatterned sample shows flat quantized plateaus at multiple integers of $2 e^{2} / h$. This indicates the formation of a ballistic quantum point contact $^{8}$ formed by an adiabatic potential without any impurities. The appearance of the conductance plateaus demonstrates that the grooves scribed with the diamond tip define a smooth potential without significant fluctuations. In contrast, the conductance of the silicon-patterned sample, shown in Fig. 2(b), exhibits only a few poorly resolved conductance plateaus.

For further characterization of the diamond-patterned sample we applied an additional dc source-drain bias voltage $V_{\mathrm{SD}}$ that allows us to determine the 1D-subband spacing. ${ }^{9}$ The transconductance $d G / d V_{I P G}$ derived numerically from these measurements is plotted as a function of $V_{I P G}$ and $V_{\mathrm{SD}}$ in the gray scale plot in Fig. 3(a). The corresponding conductance is given by the numbers inside the dark regions and the diamonds for $G=4\left(2 e^{2} / h\right)$ and $G=5\left(2 e^{2} / h\right)$ are marked with dashed lines. The crossing of adjacent zero-bias peaks $N$ and $N+1$ at finite bias $e V_{\mathrm{SD}}$ reveals the energy spacing $\quad \Delta E_{N, N+1}=e V_{\mathrm{SD}}$ ranging from $\Delta E_{2,3}$ $=2.5( \pm 0.1) \mathrm{meV}$ for the second and third subbands to $\Delta E_{4,5}=2.3( \pm 0.1) \mathrm{meV}$. Whereas the subband spacing in split gate devices at higher subband indices drastically decreases we observe only a slight reduction for our sample at $N>1$. This indicates that the shape of confinement inside the constriction remains nearly unaffected by the gate voltage and is only shifted up and down. Assuming a harmonic confinement potential and a gate voltage dependent potential 
barrier we deduce a value of $w \sim 160 \mathrm{~nm}$ for the electronic width of the constriction at zero gate voltage with $\sim 5$ occupied subbands. The depletion length for the diamond tip then can be determined to $w_{\text {depl }} \sim 180 \mathrm{~nm}$ which nearly is half the length of $w_{\mathrm{depl}} \sim 330 \mathrm{~nm}$ extracted for the silicon-patterned sample. The larger depletion length of the silicon tip as well as the creation of significant potential fluctuations are probably related to an enhanced formation of surface defects caused by the increased number of scans.

By inspection of the first conductance step in the righthand side inset of Fig. 2(a) we observe an additional shoulder close to $0.7\left(2 e^{2} / h\right)$. In the gray scale plot in Fig. 3(a) this shoulder leads to additional plateaus for finite bias voltages at $G<2 e^{2} / h$ marked with arrows. This can be seen more clearly in Fig. 3(b), where we plotted $G=d I / d V_{\mathrm{SD}}$ as a function of dc source-drain voltage taken at fixed gate voltages. Whereas the majority of the plateaus appear at multiples of $2 e^{2} / h$, below $2 e^{2} / h$ extra plateaus appear at $0.3\left(2 e^{2} / h\right)$ and $0.7\left(2 e^{2} / h\right)$ marked by the horizontal dashed line. The so-called 0.7 anomaly $^{10}$ is an indicator for very clean one-dimensional channels and is considered to be caused by electron-electron interactions. The exact underlying mechanism of this structure is still not clarified but it is an intrinsic property of low-disorder quantum point contacts. Together with the well-resolved plateaus the appearance of the 0.7 anomaly shows that we scribed an adiabatic-like constriction free from significant potential fluctuations with the diamond tip.

In conclusion, we fabricated quantum point contact devices by engraving a constriction into a GaAs/AlGaAs heterostructure with the tip of an atomic force microscope. To study the influence of the tip material we engraved devices using both a silicon tip and a diamond tip. It turned out that a diamond tip is almost perfect not only on the basis of a fast and simple processing but also in forming proper potential profiles to observe ballistic electron transport. The appearance of the $0.7\left(2 e^{2} / h\right)$ conductance anomaly confirms the high quality of diamond-engraved devices. We deduced the depletion lengths induced by the different tips yielding $w_{\text {depl }} \sim 180 \mathrm{~nm}$ for diamond-engraved samples which is roughly two times smaller than typical depletion lengths in silicon-patterned devices.

The authors thank P. Hullmann for his assistance with the scanning electron microscope. This work was supported by the BMBF.

\footnotetext{
${ }^{1}$ See, e.g., A. Fuhrer, S. Lüscher, T. Ihn, T. Heinzel, K. Ensslin, W. Wegscheider, and M. Bichler, Nature (London) 413, 822 (2001).

${ }^{2}$ H. W. Schumacher, U. F. Keyser, U. Zeitler, R. J. Haug, and K. Eberl, Appl. Phys. Lett. 75, 1107 (1999).

${ }^{3}$ R. Magno and B. R. Bennett, Appl. Phys. Lett. 70, 1855 (1997).

${ }^{4}$ J. Cortes Rosa, M. Wendel, H. Lorenz, J. P. Kotthaus, M. Thomas, and H. Kroemer, Appl. Phys. Lett. 73, 2684 (1998).

${ }^{5}$ C. K. Hyon, S. C. Choi, S.-H. Song, S. W. Hwang, M. H. Son, D. Ahn, Y. J. Park, and E. K. Kim, Appl. Phys. Lett. 77, 2607 (2000).

${ }^{6}$ Tapping-mode probes made by Nanosensors.

${ }^{7}$ A. Malavé, K. Ludolph, T. Leinhos, Ch. Lehrer, L. Frey, and E. Oesterschulze, Appl. Phys. A. (in press).

${ }^{8}$ See, e.g., C. W. J. Beenaaker and H. van Houten, in Quantum Transport in Semiconductor Nanostructures, Solid State Physics (Academic, New York, 1991), Vol. 44.

${ }^{9}$ N. K. Patel, J. T. Nicholls, L. Martín-Moreno, M. Pepper, J. E. F. Frost, D. A. Richtie, and G. A. C. Jones, Phys. Rev. B 44, 13549 (1991).

${ }^{10}$ K. J. Thomas, J. T. Nicholls, M. Y. Simmons, M. Pepper, D. R. Mace, and D. A. Richtie, Phys. Rev. Lett. 77, 135 (1996).
} 